\title{
Association of Reduced Tract Integrity with Social Communication Deficits in Preschool Autism Children: A Tract-Based Spatial Statistics Study
}

\author{
Yi Yin ${ }^{1,2, *}$ \\ Shoujun $\mathrm{Xu}^{3, *}$ \\ Chao $\mathrm{Li}^{4}$ \\ Meng $\mathrm{Li}^{2}$ \\ Mengchen Liu $^{2}$ \\ Jianhao Yan $^{2}$ \\ Zhihong Lan ${ }^{1,2}$ \\ Wenfeng Zhan ${ }^{2}$ \\ Guihua Jiang (D) 1,2 \\ Junzhang Tian ${ }^{1,2}$ \\ 'The Second School of Clinical Medicine, \\ Southern Medical University, Guangzhou, \\ People's Republic of China; ${ }^{2}$ Department \\ of Medical Imaging, Guangdong Second \\ Provincial General Hospital, Guangzhou, \\ People's Republic of China; ${ }^{3}$ Department \\ of Radiology, Shenzhen Children's \\ Hospital, Shenzhen, People's Republic of \\ China; ${ }^{4}$ Department of Radiology, The \\ First Affiliated Hospital of China Medical \\ University, Shenyang, People's Republic of \\ China
}

*These authors contributed equally to this work
Correspondence: Junzhang Tian; Guihua Jiang

Department of Medical Imaging, Guangdong Second Provincial General Hospital, No.466, Xingang Road, Guangzhou, China

Tel +8620 89I6870I; +86 I8620I20333 Email tianjunzhang20I5@I63.com; GH. jiang2002@163.com
Purpose: To analyze the changes in white matter tracts in preschool children with autism spectrum disorder (ASD), and the correlation between these changes and social communication deficits.

Methods: Diffuse tensor imaging was used to assess white matter integrity using tract-based spatial statistics in a sample of 50 right-handed children with ASD aged 2-6 years vis a reference sample of 46 typically developing children aged 2-6 years. We then correlated these significant different fiber tracts between groups with communication and social interaction scores using the Autism Diagnostic Interview-Revised Assessment (ADI-R) Scale.

Results: We observed decreased fractional anisotropy (FA) in tracts including the left superior longitudinal fasciculus (SLF), the splenium of the corpus callosum (splCC), the left corticospinal tracts, and the left inferior longitudinal fasciculus (ILF) in children with ASD. Specifically, there was reduced white matter integrity of these tracts in the left cerebral hemisphere. In addition, we found that the decreased FA of left SLF and ILF was negatively associated with the ADI-R scores in children with ASD.

Conclusion: The structural integrity of some white matter tracts in the five-level anatomical model for the social communication was reduced. The reduced integrity white matter that we observed primarily in the left cerebral hemisphere may be a neural substrate of social communication deficits in preschool children with ASD, and we speculate that the reduction is associated with the severity of social interaction. The reduced FA of the splCC might be a substantial biomarker for children with ASD.

Keywords: autism spectrum disorder, child \& adolescent psychiatry, diffuse tensor image, neuroimaging in psychiatry, tract-based spatial statistics

\section{Introduction}

Autism spectrum disorder (ASD) is a neurodevelopmental disorder that begins in infants and young children. ${ }^{1}$ Its core symptoms include social interaction and communication deficits, limited interests, and repetitive stereotyped behaviors. ${ }^{2}$

It was reported that ASD affects as many as 1 in 54 children in the USA in $2016 .^{3}$ There is also a high prevalence rate in China; the number of children with ASD in China may reach more than 3 million, ${ }^{4}$ which results in a significant burden to family and society.

The neural substrates of ASD remain unclear, and some evidence suggests that ASD is a neurodevelopmental disorder caused by the interaction of various biological and environmental factors. ${ }^{5}$ In recent years, more and more ASD research has focused 
on neuroimaging, especially in diffuse tensor imaging (DTI). This technique provides a powerful means to facilitate noninvasive clinical diagnostics of various neural system diseases and abnormalities. Tract-based spatial statistics (TBSS) is considered an effective method used in DTI to assess white matter in a voxel-wise approach without the necessity of a priori hypotheses (Smith, 2006). ${ }^{6,7}$ Thus, it may be particularly useful for identifying WM (white matter) neurodevelopmental alterations in ASD. Directiondependent diffusivity of water molecules is reflected in fractional anisotropy (FA), a quantitative index which is sensitive to WM integrity. The development of WM in typically developed children will undergo a process of myelination, ${ }^{8}$ while some studies show that ASD children suffer from abnormal white matter development; indeed, a previous study found reduced integrity of posterior fiber tracts in children with ASD aged 8-12 years. ${ }^{9}$ Researchers have proposed tract-specific patterns of WM abnormalities associated with ASD in children and adolescents aged 9-20. ${ }^{10}$ In addition, one study using DTI in an ASD model of monkeys suggested that the decreased size of anterior commissure in neonatal monkeys may be linked to social dysfunction during adulthood, ${ }^{11}$ suggesting that the reduced integrity of specific tracts may lead to social communication deficits.

Catani and Bambini (2014) proposed a hypothesis of the five-level anatomical model for the social communication. $^{12}$ This hierarchical model in relation to developmental and evolutionary trajectories includes the superior longitudinal fasciculus (SLF) for informative actions (level 1), the frontal aslant tracts (FAT) for communicative intentions (level 2), the uncinate fasciculus (UF), inferior longitudinal fasciculus (ILF), and inferior frontal-occipital fasciculus (IFOF) for lexical and semantic processing (level 3), the arcuate fasciculus (AF) for syntactic analysis (level 4), and the temporal-parietal tracts (TPT) for pragmatic integration (level 5). This model provides a better understanding of the structural correlates of the model for social communication. Some research suggests the social communication deficits in ASD may be caused by reduced integrity of WM; ${ }^{13-15}$ however, most studies focused on adolescents or adults with ASD. The early years of life mark an important period for the development of WM, and this period is critical for brain development and present a target for early intervention. ${ }^{16}$ However, the relationship between WM integrity and social communication deficits in preschool ASD children aged 2-6 years remains unclear.
Our study aims to clarify the relationship between WM integrity and social communication deficit in children with ASD aged 2-6 years. In assessing WM integrity in children aged 2-6 years with ASD compared to typically developing (TD) children, we sought to identify whether WM integrity in ASD children is compromised. Finally, we investigated the relationship between social communication deficits and WM integrity.

\section{Materials and Methods \\ Participants}

Participants were recruited from the Shenzhen Children's Hospital. Inclusion criteria for ASD group were (1) two psychiatrists with associate director and above jointly diagnosed the child according to the DSM-V ASD diagnostic criteria; (2) 2-6 years old; (3) right-handed male or female, and inclusion criteria for control group (1) 2 to 6 years old; (2) right-handed male or female; (3) typical development; (4) diagnostic criteria for exclusion of ASD by scale evaluation. Common exclusion criteria were (1) a history of serious mental illnesses, trauma or epilepsy, neurological diseases, and major physical diseases; a history of taken antipsychotic drugs; (2) those who did not cooperate or could not effectively complete the MRI examination during the test. We use foam cushions between the headphones and the head coil to minimize motion artifacts. We excluded eight subjects due to head motion as well as low image quality. In total, 50 ASD children and 46 age- and sex-matched TD children took part in the study.

The Committee on Medical Ethics of Southern Medical University, The Second School of Clinical Medicine Institutional Review Board approved this study, which was carried out in accordance with the Declaration of Helsinki. Written informed consent was obtained after the parents of participants were given a complete description of the study.

\section{MRI Acquisition and Pre-Processing}

Participants were scanned using a 3.0T Siemens Skyra scanner in the Department of Radiology, Shenzhen Children's Hospital. All subjects were sedated with 10\% chloral hydrate. The participants' heads were fixed with a sponge pad, wore earplugs to attenuate noise. Anatomical high-resolution structural MR images were obtained using a T1-weighted three-dimensional spoiled gradient recalled pulse sequence. T1 acquisition 
parameters: $\mathrm{TR}=2300 \mathrm{~ms}, \mathrm{TE}=2.26 \mathrm{~ms}$, flip angle $=8^{\circ}$, acquisition matrix $=256 \times 256, \mathrm{FOV}=256 \mathrm{~mm} \times$ $256 \mathrm{~mm}$, and $1.00-\mathrm{mm}$ slice thickness with a $0.5-\mathrm{mm}$ inter-slice gap; DTI acquisition parameters: TR/TE = $11,400 / 85 \mathrm{~ms}, \mathrm{~b}=700 \mathrm{~s} / \mathrm{mm}^{2}$, voxel size $=2 \times 2 \times 2 \mathrm{~mm}$, $\mathrm{FOV}=256 \times 256 \mathrm{~mm}$, acquisition matrix $=128 \times 128$, slices $=75$.

DTI data analysis was carried out using the toolkit in FSL (FMRIB Software Library, http://www.finrib.OX.ae. $\underline{\mathrm{uk} / \mathrm{fs} \mathrm{l}}$ ). We took the following steps in processing the data: (1) converted all the original dicom data of the DTI into nifti format; (2) performed motion and eddy current correction; used the DTIFIT tool to calculate the fractional anisotropy (FA) map, and used FSL Brain Extraction Tool (BET) to extract brain tissue; (3) aligned participants' FA images into a target image using the nonlinear tool FNIRT; the target image was generated by aligning every FA image to one another to identify the most representative image; (4) affine-aligned this image into the JHU ICBM-DTI-81 atlas space; (5) calculated the mean of all FA images and thinned to create a mean FA skeleton; to exclude gray matter or CSF, we used a threshold of 0.2 for the creation of the mean FA skeleton, and each participant's aligned FA image was then projected onto this skeleton; (6) performed a nonparametric two-sample permutation test (5000 permutations) to assess the group-related differences using threshold-free cluster enhancement (TFCE); for statistical inference, family-wise error (FWE) correction was performed and the significance threshold was set to $p<$ 0.05 for multiple comparisons; (7) the filled result data were generated using the script "tbss_fill" provided in FSL.

\section{Assessment for Neurocognition and Social Function}

The participants underwent neuropsychological test for autism traits as well as social/communication performance, the neuropsychological test include the Autism Behavior Checklist (ABC), ${ }^{17}$ the Childhood Autism Rating Scale (CARS), ${ }^{18}$ and the Autism Diagnostic Interview-Revised Assessment Scale (ADI-R), ${ }^{19}$ the Pediatric Examination Table of Neuropsychological Development (Institute of Pediatrics of Capital Medical University, CNBSR 2016) was used to assess the Development Quotient (DQ).

\section{Statistical Analyses}

We used SPSS21.0 to analyze the data. The sex of the two groups was compared using the chi-square test, and age was compared using an independent sample $t$-test. We used Pearson correlation analyses to compare FA values with the scores of the behavioral measures in order to explore the relationship between the abnormal WM integrity in children with ASD and the associated clinical manifestation behaviors. Age and sex were taken as covariates, and the significance level was set to $p<0.05$ with applied FDR corrections for multiple comparisons.

\section{Results}

\section{Demographic Characteristics}

The 50 participants in the ASD group had an average age of $3.26 \pm 1.2$ years, and the 46 TD participants in the control group had an average age of $3.47 \pm 1.5$ years. The $\mathrm{ABC}, \mathrm{CARS}$, and ADI-R scores in the ASD group were significantly higher than those in the TD group $(p<0.05)$, while the DQ scores were significantly lower $(p<0.05)$. There were no significant differences between the ASD and TD groups in age, gender (Table 1).

\section{Comparison of FA Values of White Matter Tract Between the ASD Group and the TD Group}

Compared to the TD group, the left superior longitudinal fasciculus (SLF), the left corticospinal tract (CT), the left Inferior longitudinal fasciculus (ILF), and the splenium of the corpus callosum (splCC) were significantly reduced in

Table I Demographic and Clinical Characteristics of Participants

\begin{tabular}{|l|l|l|l|}
\hline & ASD $(\mathbf{n}=\mathbf{5 0})$ & TD $(\mathbf{n}=\mathbf{4 6})$ & $\mathbf{p}$-value \\
\hline Age (years) & $3.26 \pm 1.58$ & $3.47 \pm 1.65$ & 0.459 \\
\hline Sex (M/F) & $43 / 7$ & $38 / 8$ & 0.076 \\
\hline CARS & $35.16 \pm 3.82$ & $18.46 \pm 3.26$ & $<0.05^{*}$ \\
\hline ABC & $68.04 \pm 9.43$ & $37.74 \pm 8.63$ & $<0.05^{*}$ \\
\hline ADI-R (COM) & $13.31 \pm 3.93$ & $10.23 \pm 3.42$ & $<0.05^{*}$ \\
\hline ADI-R (SOC) & $20.92 \pm 2.20$ & $14.31 \pm 2.32$ & $<0.05^{*}$ \\
\hline DQ & $69.75 \pm 5.66$ & $105.72 \pm 6.76$ & $<0.05^{*}$ \\
\hline
\end{tabular}

Note: Results are expressed as the mean \pm SD, ${ }^{*} p<0.05$.

Abbreviations: CARS, the Childhood Autism Rating Scale; $A B C$, the Autism Behavior Checklist; ADI-R (SOC), the Autism Diagnostic Interview-Revised Social Scores; ADI-R (COM) the Autism Diagnostic Interview-Revised Communication Scores; DQ, Development Quotient. 
ASD children (TFCE FWE corrected, $p<0.05$ ), and no FA value increase was found in the ASD group (Table 2) (Figure 1).

\section{Correlation Analysis Between FA Value of White Matter Tract and CARS Scores with the Two Groups}

Decreased FA was found in children with ASD in several tracts including the splCC, left CT, left ILF, and left SLF. A negative correlation between FA values and CARS scores was observed in children with ASD in the splCC ( $\mathrm{r}=-0.897, p<0.001$, FDR corrected) (Figure 2). The ADI-R scores in the communicative and social domains were also negatively correlated with the FA values in the left ILF and left SLF (Table 3) (Figures 3 and 4).

\section{Discussion}

In this study, we investigated the WM integrity and its association with social communication and social interaction functions in children with ASD. The decreased FA was found mainly in several tracts including the splCC, left CT, left ILF, and left SLF in children with ASD. These findings suggest WM microstructural damage in ASD children. Furthermore, the negative correlation between FA values and CARS scores was observed in children with ASD in the splCC. Finally, we found that decreased FA of left SLF and ILF were negatively associated with ADI-R scores.

According to the five-level anatomical model for the social communication proposed by Catani and Bambini, ${ }^{12}$ the SLF is relevant for informative actions (level 1) as the first step of social interaction, while the ILF relates to lexical and semantic processing (level 3). Our study

Table 2 Regions with Significant $(p<0.05$, Corrected for Multiple Comparisons) Reduced Fractional Anisotropy (FA) in Children with ASD vs TD

\begin{tabular}{|l|c|c|c|}
\hline & \multicolumn{3}{|c|}{ MNI Coordinates } \\
\hline & $\mathrm{x}$ & $\mathrm{y}$ & $\mathrm{z}$ \\
\hline Inferior longitudinal fasciculus (L) & -41 & -25 & -4 \\
\hline Superior longitudinal fasciculus (L) & -36 & -19 & 29 \\
\hline Corticospinal tract (L) & -26 & -20 & -14 \\
\hline Splenium of corpus callosum (L) & -19 & -37 & 30 \\
\hline Splenium of corpus callosum (R) & 16 & -35 & 30 \\
\hline
\end{tabular}

suggests that the reduced integrity of the left SLF and ILF may affect the first and third level of the five-level anatomical model for the social communication, leading to social communication deficits in children with ASD.

We further observed that the FA values in the left ILF were negatively correlated with ADI-R communicative domains. According to the five-level anatomical model for the social communication hypothesis, the ILF is relevant for for lexical and semantic processing (level 3). The ILF is a major occipitotemporal association tract that connects the ventral surface of the anterior temporal lobe and the extrastriate cortex of the occipital lobe, ${ }^{20}$ and research has shown the left ILF plays an important role in language and semantic processing. ${ }^{21}$ In children with ASD, reduced integrity of the left ILF may lead to a failed understanding of other's verbal expressions resulting in poor communication with others. Research has also identified the ILF as being relevant to emotion management, ${ }^{22}$ in which the decreased FA of the left ILF may affect facial expression recognition and emotion perception, subsequently affecting social communication abilities.

In addition, we found that the FA values in the left SLF were correlated with the ADI-R (SOC). The SLF is an important and one of the longest association tracts connects the temporal, frontal, and parietal lobe, which are responsible for advanced cognitive functions. The SLF transfers somatosensory information between the ventral prefrontal cortex, the supramarginal gyrus, and the lateral inferior prefrontal cortex, ${ }^{23}$ and its location in the frontalparietal network mirror neuron system provides support for sensorimotor integration. ${ }^{24}$ This area may also convey relevant information from others and elicit actions from others, which is a key aspect of social interaction. ${ }^{25}$ Our study suggests that compromised integrity of the left SLF may lead to social communication deficits in children with ASD.

However, according to the five-level model hypothesis, we did not find a significant difference in the FA values of the tracts at the frontal aslant tracts (FAT) (level 2), arcuate fascicle (level 4) and temporoparietal tract (level 5) between ASD and TD groups. The arcuate fascicle (AF) connects the frontal, temporal, and parietal cortex areas. Its direct pathway, called the classical pathway as it connects the temporal cortex and the prefrontal cortex, is related to speech production. The indirect pathway travels between the inferior parietal, Broca's area, and the posterior part of the temporal parietal lobe, which are associated with language comprehension. We found no significant 




Figure I Sagittal (panels A, B), coronal (panels C, D), and horizontal (panels E, F) sections showing areas of significantly decreased FA (P < 0.05 , corrected) in ASD compared to age-matched controls, displayed on the MNI template brain. Regions of decreased FA in ASD are highlighted on the mean FA skeleton (green) in colored voxels (scale ranging from red to yellow). For better visualization, the stats images are "thickened" with tbss_fill.

Abbreviations: CT, corticospinal tract; ILF, inferior longitudinal fasciculus; SLF, superior longitudinal fasciculus; spICC, splenium of corpus callosum.

difference between the two fiber tracts between ASD and TD children. One possible explanation for this finding is that the arcuate and temporoparietal tracts mature in later childhood, and the younger age of our participants may have not allowed us to observe this maturation yet.

Furthermore, the corpus callosum (CC) plays an important role in the lateral asymmetry of brain function ${ }^{26-29}$ as the main transverse fibers between bilateral cerebral hemispheres. Abnormal development of the CC may affect the integration and communication of information between the hemispheres, affecting cognitive function in ASD. Specifically, this damage may be related to the decrease in $\mathrm{CC}$ axons in children with $\mathrm{ASD}{ }^{30}$ In this study, we observed reduced FA values in the splCC in children with ASD, which communicates somatosensory information between the bilateral parietal lobe and the occipital lobe. ${ }^{31}$ The parietal cortex, which is closely related to various deep and shallow sensations, has fiber tracts projected to the splCC, which may explain why some children with ASD often have the symptom of paresthesia; here, we also found a negative correlation between the splCC and CARS scores. Abnormalities in the CC in ASD have also been observed in other studies. One such study suggested that the reduced $\mathrm{CC}$ integrity may be related to nonverbal

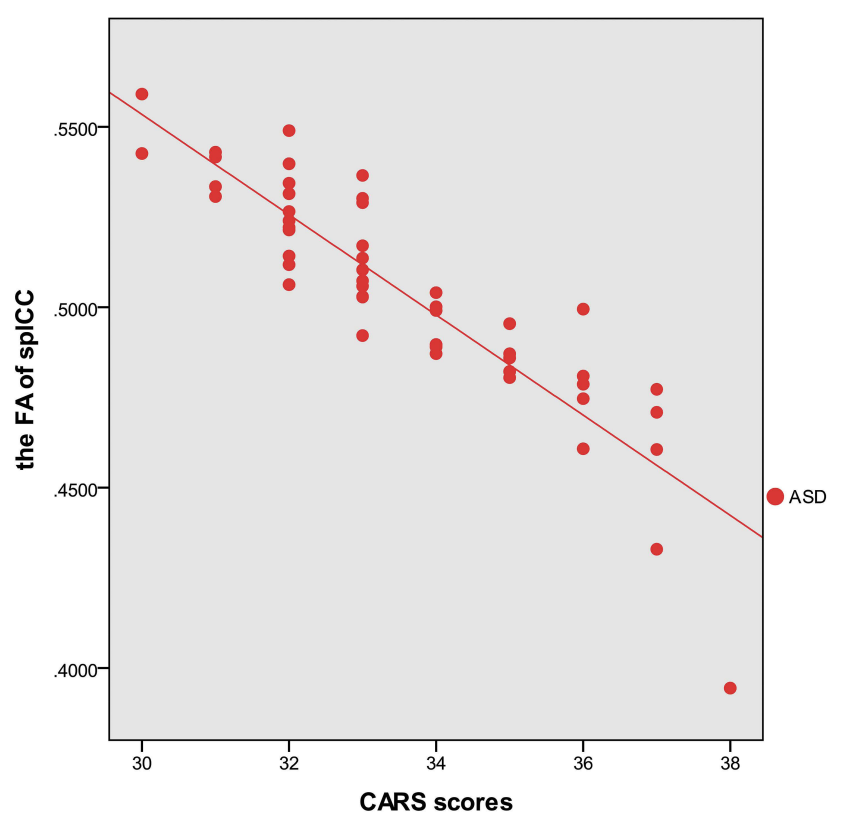

Figure 2 Correlations between the FA of splenium of the corpus callosum and CARS scores in ASD children aged $2-6$ years $(r=-0.89, p<0.001)$. 
Table 3 Partial Correlation Analysis Controlling for Age to Assess the Relationships Between the Reduced FA of WM and the ADI-R (COM), and ADI-R (SOC) Within the ASD Group

\begin{tabular}{|l|l|l|}
\hline & ADI-R (COM) & ADI-R (SOC) \\
\hline $\begin{array}{l}\text { Inferior longitudinal } \\
\text { fasciculus (L) }\end{array}$ & $r=-0.789, p<$ & $r=-0.1 \mathrm{II}, p=$ \\
$0.00 I^{*}$ & 0.528 \\
\hline $\begin{array}{l}\text { Superior longitudinal } \\
\text { fasciculus (L) }\end{array}$ & $r=-0.206, p=$ & $\begin{array}{l}r=-0.730, p< \\
0.00 I^{*}\end{array}$ \\
\hline Corticospinal tract (L) & 0.245 & $r=-0.068, p=-0.007, p=$ \\
& 0.712 & 0.934 \\
\hline Splenium of corpus & $r=-0.053, p=$ & $r=-0.047, p=$ \\
callosum & 0.734 & 0.732 \\
\hline
\end{tabular}

Note: *Significant difference after FDR correction, $p<0.001$.

Abbreviations: ADI-R (SOC), the Autism Diagnostic Interview-Revised Social Scores; ADI-R (COM), the Autism Diagnostic Interview—Revised Communication Scores.

cognitive performance, ${ }^{32}$ and that these reductions observed in ASD persists into adulthood. ${ }^{33}$ We also observed a negative correlation between FA values and CARS scores in the splCC of children with ASD, suggesting that reduced FA in this region might be a substantial biomarker early detection of ASD.

In addition, we found reduced FA value in the left $\mathrm{CT}$, which is a tract for voluntary motor control of the body and limbs. The participants in our study also

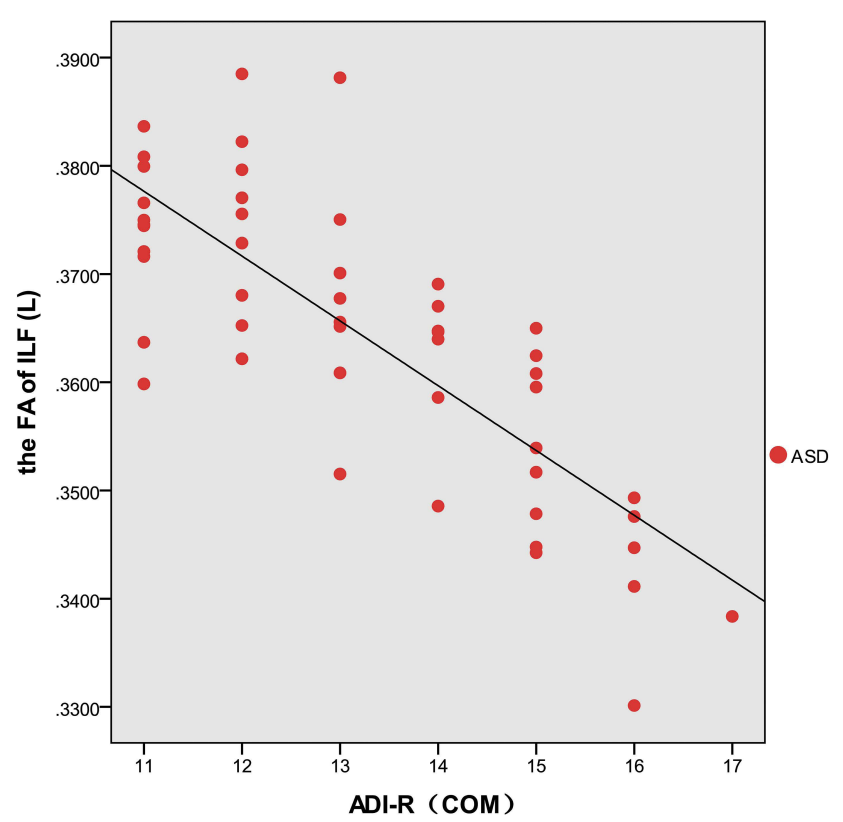

Figure 3 Correlations between the FA of ILF ( $L$ ) and ADI-R communication scores in ASD children aged $2-6$ years $(r=-0.78, p<0.001)$. showed many repetitive stereotyped behaviors, often referred to as "being clumsy". ${ }^{34}$ However, our study indicates that reduced WM integrity in left CT may be related to these behaviors in preschool children with ASD.

We found almost all the reduced FA tracts were in the left, rather than the right, hemisphere among ASD children. This may suggest that tract development in ASD children has rightward asymmetry. A previous study also found both ASD toddlers and adults had widespread rightward asymmetry, in both gray and white matter. ${ }^{35}$ As the left hemisphere of most TD right-handed individuals is specialized for language and motor control, ${ }^{36}$ our findings suggest that atypical lateralization in children with ASD may also lead to their observed social communication deficits.

\section{Limitations}

Our study has several limitations. First, our study was cross-sectional and all participants were preschool children aged 2-6 years. Therefore, the conclusions cannot be universally extended to other types of ASD patients, necessitating future longitudinal datasets. Moreover, although almost all reduced FA of WM was observed in the left hemisphere, we observed instances in the right hemisphere across groups. The significance of these findings should be explored in a future study.

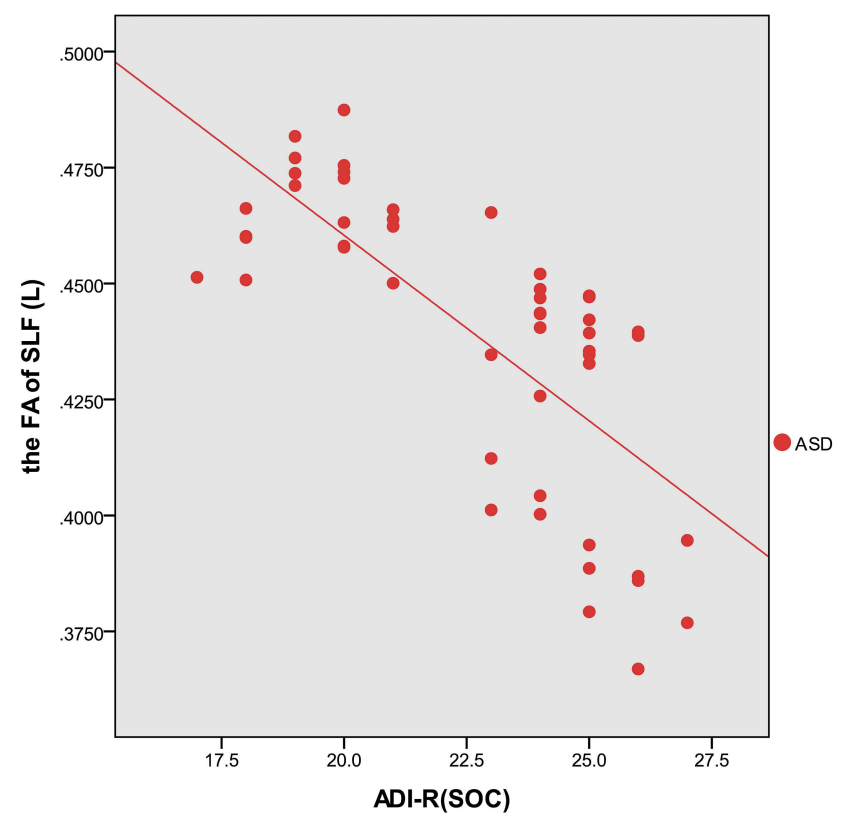

Figure 4 Correlations between the FA of SLF (L) and ADI-R social scores in ASD children aged $2-6$ years $(r=-0.73, p<0.00 \mathrm{I})$. 
In conclusion, our study identified the integrity of the left tracts is damaged in preschool children. The integrity of the splCC might be a potential biomarker for identifying ASD children aged 2-6 years. The WM integrity of the five-level anatomical model for the social communication (level 1-3) in preschool ASD children is damaged, the reduced integrity in these tracts (level 1-3) is associated with the severity of social interaction.

\section{Acknowledgments}

The authors thank all the participants and staff involved in this study. This study was supported by the National Natural Science Foundation of China (Grant Nos. 81701111 and 81771807) and the Sanming project of Medicine in Shenzhen (Grant Nos. SZSM 202011005 and SZSM 201612036)

\section{Author Contributions}

All authors made substantial contributions to conception and design, acquisition of data, or analysis and interpretation of data; took part in drafting the article or revising it critically for important intellectual content; agreed to submit to the current journal; gave final approval of the version to be published; and agree to be accountable for all aspects of the work.

\section{Disclosure}

The authors declare no conflicts of interest.

\section{References}

1. Ouyang M, Cheng H, Mishra V, et al. Atypical age-dependent effects of autism on white matter microstructure in children of 2-7 years. Hum Brain Mapp. 2016;37(2):819-832. doi:10.1002/hbm.23073

2. Kanner L. Autistic disturbances of affective contact. Acta Paedopsychiatr. 1968;35(4):100-136.

3. Maenner MJ, Shaw KA, Baio J, et al. Prevalence of autism spectrum disorder among children aged 8 years - autism and developmental disabilities monitoring network, 11 sites, United States, 2016. MMWR Surveill Summ. 2020;69(4):1-12.

4. Wang F, Lu L, Wang SB, et al. The prevalence of autism spectrum disorders in China: a comprehensive meta-analysis. Int J Biol Sci. 2018;14(7):717-725.

5. Rossignol DA, Genuis SJ, Frye RE. Environmental toxicants and autism spectrum disorders: a systematic review. Transl Psychiatry. 2014;4(2):e360.

6. Li Y, Zhou Z, Chang C, et al. Anomalies in uncinate fasciculus development and social defects in preschoolers with autism spectrum disorder. BMC Psychiatry. 2019;19(1):399.

7. Smith SM, Jenkinson M, Johansen-Berg H, et al. Tract-based spatial statistics: voxelwise analysis of multi-subject diffusion data Neuroimage. 2006;31(4):1487-1505.

8. Stassart RM, Möbius W, Nave KA, Edgar JM. The Axon-Myelin Unit in Development and Degenerative Disease. Front Neurosci. 2018;12:467.
9. Payabvash S, Palacios EM, Owen JP, et al. White matter connectome edge density in children with autism spectrum disorders: potential imaging biomarkers using machine-learning models. Brain Connect. 2019;9(2):209-220.

10. Shukla DK, Keehn B, Müller RA. Tract-specific analyses of diffusion tensor imaging show widespread white matter compromise in autism spectrum disorder. J Child Psychol Psychiatry. 2011;52 (3):286-295.

11. Mimura K, Oga T, Sasaki T, et al. Abnormal axon guidance signals and reduced interhemispheric connection via anterior commissure in neonates of marmoset ASD model. Neuroimage. 2019;195:243-251.

12. Catani M, Bambini V. A model for Social Communication And Language Evolution and Development (SCALED). Curr Opin Neurobiol. 2014;28:165-171.

13. Blanken L, Muetzel RL, Jaddoe V, et al. White matter microstructure in children with autistic traits. Psychiatry Res Neuroimaging. 2017;263:127-134.

14. Kato Y, Kagitani-Shimono K, Matsuzaki J, et al. White matter tract-cognitive relationships in children with high-functioning autism spectrum disorder. Psychiatry Investig. 2019;16(3):220-233.

15. Li SJ, Wang Y, Qian L, et al. Alterations of white matter connectivity in preschool children with autism spectrum disorder. Radiology. 2018;288(1):209-217.

16. Casey BJ, Tottenham N, Liston C, Durston S. Imaging the developing brain: what have we learned about cognitive development? Trends Cogn Sci. 2005;9(3):104-110.

17. Krug DA, Arick J, Almond P. Behavior checklist for identifying severely handicapped individuals with high levels of autistic behavior. J Child Psychol Psychiatry. 1980;21(3):221-229.

18. Schopler E, Reichler RJ, DeVellis RF, Daly K. Toward objective classification of childhood autism: childhood Autism Rating Scale (CARS). J Autism Dev Disord. 1980;10(1):91-103.

19. Lord C, Pickles A, McLennan J, et al. Diagnosing autism: analyses of data from the Autism Diagnostic Interview. J Autism Dev Disord. 1997;27(5):501-517.

20. Catani M, Jones DK, Donato R, Ffytche DH. Occipito-temporal connections in the human brain. Brain. 2003;126(Pt 9):2093-2107.

21. Mandonnet E, Nouet A, Gatignol P, Capelle L, Duffau H. Does the left inferior longitudinal fasciculus play a role in language? A brain stimulation study. Brain. 2007;130(3):623-629.

22. Wang Y, Metoki A, Smith DV, et al. Multimodal mapping of the face connectome. Nat Hum Behav. 2020;4(4):397-411.

23. Makris N, Kennedy DN, McInerney S, et al. Segmentation of subcomponents within the superior longitudinal fascicle in humans: a quantitative, in vivo, DT-MRI study. Cereb Cortex. 2005;15 (6):854-869.

24. Rizzolatti G, Sinigaglia C. The functional role of the parieto-frontal mirror circuit: interpretations and misinterpretations. Nat Rev Neurosci. 2010;11(4):264-274.

25. Lo YC, Chen YJ, Hsu YC, Tseng WI, Gau SS. Reduced tract integrity of the model for social communication is a neural substrate of social communication deficits in autism spectrum disorder. $J$ Child Psychol Psychiatry. 2017;58(5):576-585.

26. Ohta H, Aoki YY, Itahashi T, et al. White matter alterations in autism spectrum disorder and attention-deficit/hyperactivity disorder in relation to sensory profile. Mol Autism. 2020;11(1):77.

27. Hattori A, Kamagata K, Kirino E, et al. White matter alterations in adult with autism spectrum disorder evaluated using diffusion kurtosis imaging. Neuroradiology. 2019;61(12):1343-1353.

28. Giuliano A, Saviozzi I, Brambilla P, Muratori F, Retico A, Calderoni S. The effect of age, sex and clinical features on the volume of Corpus Callosum in pre-schoolers with Autism Spectrum Disorder: a case-control study. Eur J Neurosci. 2018;47(6):568-578.

29. Sui YV, Donaldson J, Miles L, Babb JS, Castellanos FX, Lazar M. Diffusional kurtosis imaging of the corpus callosum in autism. Mol Autism. 2018;9:62. 
30. Lazar M, Miles LM, Babb JS, Donaldson JB. Axonal deficits in young adults with High Functioning Autism and their impact on processing speed. Neuroimage Clin. 2014;4:417-425.

31. Zakharova NE, Potapov AA, Kornienko VN, et al. [Assessment of brain neural pathways in diffuse axonal injuries using diffusion-tensor magnetic resonance tomography]. Zh Vopr Neirokhir Im N N Burdenko. 2010;2:3-9; discussion 9. Russian.

32. Alexander AL, Lee JE, Lazar M, et al. Diffusion tensor imaging of the corpus callosum in Autism. Neuroimage. 2007;34(1):61-73.

33. Keller TA, Kana RK, Just MA. A developmental study of the structural integrity of white matter in autism. Neuroreport. 2007;18 (1):23-27.
34. Brito AR, Vasconcelos MM, Domingues RC, et al. Diffusion tensor imaging findings in school-aged autistic children. J Neuroimaging. 2009;19(4):337-343.

35. Fu L, Wang Y, Fang H, et al. Longitudinal study of brain asymmetries in autism and developmental delays aged 2-5Years. Neuroscience. 2020;432:137-149.

36. Gotts SJ, Jo HJ, Wallace GL, Saad ZS, Cox RW, Martin A. Two distinct forms of functional lateralization in the human brain. Proc Natl Acad Sci U S A. 2013;110(36):E3435-44.

\section{Publish your work in this journal}

Neuropsychiatric Disease and Treatment is an international, peerreviewed journal of clinical therapeutics and pharmacology focusing on concise rapid reporting of clinical or pre-clinical studies on a range of neuropsychiatric and neurological disorders. This journal is indexed on PubMed Central, the 'PsycINFO' database and CAS, and is the official journal of The International Neuropsychiatric Association (INA). The manuscript management system is completely online and includes a very quick and fair peer-review system, which is all easy to use. Visit http://www.dovepress.com/testimonials.php to read real quotes from published authors. 